\title{
ESPACIO FISCAL Y PROTECCIÓN SOCIAL EN HONDURAS $^{1}$
}

\author{
Hugo Noé Pino, Rafael Del Cid ${ }^{2}$ \\ Instituto de Investigación de Políticas Públicas, Universidad Tecnológica Centroamericana (UNITEC), \\ Campus de Tegucigalpa
}

(Recibido: Septiembre, 2017/ Aceptado: Diciembre, 2017)

\section{Resumen}

Una de las discusiones importantes en materia de política fiscal en países en vías de desarrollo es cómo generar recursos que apoyen el crecimiento económico y amplíen y mejoren el gasto social. Estas discusiones se han formalizado en la literatura económica del denominado espacio fiscal, término utilizado para referirse al esfuerzo por identificar y movilizar recursos que permitan alcanzar objetivos de crecimiento económico y/o de desarrollo social. Este trabajo presenta las principales discusiones sobre el concepto espacio fiscal, sus fuentes y experiencias recientes en algunos países. Especial énfasis tiene el análisis del caso de Honduras, tanto desde el punto de vista de lo estudiado hasta ahora, como de la revisión actual de su situación fiscal y social. Se finaliza haciendo una propuesta de los espacios fiscales que puede aprovechar Honduras para el financiamiento de la protección social.

Palabras Claves: Espacio fiscal, presupuesto público, protección social.

\begin{abstract}
One of the important discussions on fiscal policy in developing countries is how to generate resources that support economic growth and expand and improve social expending. These discussions have been formalized in the economic literature of the so-called fiscal space, a term used to refer to the effort to identify and mobilize resources to achieve economic growth and / or social development goals. This paper presents the main discussions on fiscal space, their sources and recent experiences in several countries. Especial emphasis is given to the analysis of Honduran case, both from the point of view of what has been studied up to now, and from the current revision of its fiscal and social situation. It ends by making a proposal for the fiscal spaces that Honduras could use to finance social protection.
\end{abstract}

Keywords: Fiscal space, public budget, social protection.

\footnotetext{
${ }^{1}$ El presente artículo se deriva del documento de trabajo elaborado por el Instituto de Investigación de Políticas Públicas de Unitec a solicitud de la Oficina Regional en México de la OIT, sobre el "Financiamiento de la protección social y opciones para la creación de espacio fiscal en Honduras" (en prensa). Se agradece el apoyo de Ely Maritza Noé en dicha investigación.

${ }^{2}$ Autor para correspondencia. Email: hugo.pino@unitec.edu.hn, jose.delcid@unitec.edu.hn
} 


\section{Introducción}

Después de la segunda guerra mundial, la política fiscal jugó un papel instrumental de primer orden para contribuir al crecimiento económico y lograr una mejor redistribución del ingreso en la mayoría de los países desarrollados y en vías de desarrollo. En términos simples, la política fiscal se entiende como la cantidad, forma y criterios con que un país recolecta impuestos y los gasta. En la actualidad se incluyen también dentro del estudio de la política fiscal los niveles de transparencia y rendición de cuentas, o sea, el grado en que el gobierno informa y se somete al escrutinio de la ciudadanía en el uso de los recursos públicos.

En tiempos de expansión económica se facilita observar como el crecimiento económico genera recursos para el financiamiento del gasto social. Esto suele crear la impresión errónea de que lo primero es condición necesaria (causa) de lo segundo (efecto). Por ello, en tiempos de crisis, la estabilización económica gana la mayor atención, el gasto social y la inversión pública pierden importancia y pasan a ser generalmente la parte sacrificada. La sociedad entra en la tensión entre alcanzar metas, como la reducción del déficit fiscal, y el fortalecimiento y expansión de la política social.

Se desconoce o se olvida el hecho de que los insumos del crecimiento económico no son solo el capital físico y el trabajo, en medio están, como factores condicionantes, tanto el capital humano (calidad y competencias) como el social (solidez institucional). De la calidad de estos factores dependen la innovación, el uso y la productividad del capital físico y el trabajo. Por lo mismo, para los países en desarrollo el capital humano y social deviene fundamental. Los presupuestos públicos son en cierto modo el mejor indicador de las verdaderas prioridades de un gobierno. Por ello es meritorio el escrutinio cuidadoso del presupuesto, a guiarse por el criterio de la congruencia entre objetivos sociales y recursos asignados. En este marco toma sentido la discusión sobre espacio fiscal.

Este artículo presenta en la sección siguiente las discusiones recientes sobre el concepto de espacio fiscal, con atención principal a los enfoques de "estabilidad fiscal" y de "desarrollo". El primer enfoque se atribuye al Fondo Monetario Internacional (FMI) y el segundo a las Naciones Unidas. La sección finaliza con una mención de ensayos exitosos en la búsqueda de espacio fiscal a nivel internacional. La sección tres se refiere a los esfuerzos de análisis de espacio fiscal realizados en el caso de Honduras, así como a la situación fiscal actual. Ambos aspectos sirven para establecer una propuesta de posibles fuentes de espacio fiscal para la protección social, las que se describen en la última sección a manera de conclusión.

Una primera idea intuitiva sobre el significado de espacio fiscal es que se refiere a identificación y movilización de recursos para alcanzar determinados fines. Es decir, cómo encontrar recursos de las arcas públicas para alcanzar objetivos, sean estos de crecimiento económico (p.ej. como aumentar la inversión en vías de comunicación) o de desarrollo social (p.ej. como elevar los recursos para la protección social). De lo intuitivo podemos avanzar ahora a una discusión más formal del término y de sus alcances. 


\section{El concepto de espacio fiscal: principales enfoques}

La discusión formal sobre espacio fiscal surgió inicialmente para responder a la cuestión de sí, en el marco de los acuerdos con el Fondo Monetario Internacional (FMI), los gobiernos tienen flexibilidad efectiva de encontrar "espacio" para el gasto en inversión o programas sociales que se consideran importantes.

Los enfoques principales sobre el tema de espacio fiscal se pueden considerar representados por los trabajos de Heller (2005) y de Rathin, Heuty y Letouzé $(2007)^{3}$. Heller define el espacio fiscal "como la disponibilidad presupuestaria que permite a un gobierno proveer recursos para un fin deseado sin ningún perjuicio a la sostenibilidad financiera del mismo gobierno" (2005:3). En esta definición se enfatiza la condición de que la disponibilidad de recursos y la búsqueda de espacio fiscal no provoque desequilibrios en las finanzas públicas, o sea, que aspectos como los siguientes deben ser adecuadamente considerados:

1) La búsqueda de espacio fiscal deberá prever que el aumento del gasto público en el corto plazo, y cualquier gasto futuro, pueda ser financiado con ingresos corrientes y futuros.

2) El gasto del presente tiene implicaciones futuras. Por ejemplo, la construcción de un hospital en un año determinado implica sus gastos operativos en el futuro.

3) Lo anterior requiere sustentarse en un marco fiscal de mediano plazo.

Heller también llama la atención sobre otro par de asuntos. Primero, el espacio fiscal recurrente está más limitado en los países con mayores rigidices presupuestarias; esto incluye programas recurrentes financiados con recursos externos. Segundo, el espacio fiscal en términos netos queda reducido cuando se establecen gastos que restringen otros gastos.

En suma, de acuerdo a Heller, las verdaderas medidas o políticas para crear un espacio fiscal potencialmente disponible se sitúan en dos esferas: a) el aumento del ahorro público a través de una reforma tributaria y racionalización del gasto; y b) los recursos adicionales que puedan ser movilizados de préstamos y donaciones consistentes con estabilidad macroeconómica y sostenibilidad de la deuda (2005:6). Nótese el énfasis en la estabilidad económica.

Por su parte Rathin, Heuty y Letouzé proponen un entendimiento más amplio del espacio fiscal, este es: "El financiamiento que está disponible para el gobierno como resultado de las acciones concretas de política dirigidas a mejorar la movilización de recursos, y las reformas necesarias para asegurar la gobernabilidad y el entorno económico e institucional, con el fin de que estas acciones de políticas sean eficaces en relación con el logro de un conjunto específico de objetivos de desarrollo" (2007:3). En este caso, el énfasis se pone

\footnotetext{
${ }^{3}$ Se dice representados porque estos no son los únicos autores que han tratado el tema, aunque sí los más representativos. Contrariando principios de las publicaciones citadas, uno podría decir que una es la interpretación del FMI (enfoque de la estabilidad económica) y la otra de Naciones Unidas (enfoque de desarrollo) con relación al espacio fiscal.
} 
en una movilización de recursos en simultaneidad con reformas que permitan alcanzar objetivos de desarrollo previamente acordados.

Por lo tanto, la diferencia que vale la pena destacar de estas posiciones es: La primera subraya la estabilidad fiscal como límite del espacio fiscal, mientras que la segunda enfatiza a las políticas de desarrollo como marco de tal espacio, sin que esto implique el olvido de la estabilidad; lo ideal está en que la estabilidad no constriña el accionar de las políticas públicas. Cabe la pregunta sobre la pertinencia de una tercera postura ¿En qué medida se puede hablar de una suerte de co-responsabilidad mundial para aumentar el espacio fiscal de los países en condiciones extremas de menor desarrollo? Esta responsabilidad se torna observable en la llamada asistencia oficial al desarrollo, que ofrecen los países de mayor a los de menor desarrollo bajo la forma de ayudas y alivio de deuda.

\subsection{El enfoque que enfatiza la sostenibilidad fiscal}

Las fuentes del espacio fiscal son similares para ambos enfoques, por ejemplo, Heller (2015:3) señala que las fuentes de espacio fiscal pueden provenir de:

\section{(a) Aumento de impuestos o mejoras en la administración tributaria}

Se discute el límite a que puede llegar la carga tributaria de modo que no desincentive la inversión privada. También interesa discutir la conveniencia de establecer impuestos con fines determinados siendo que estos, a su vez, reducen el espacio fiscal de otros gastos.

\section{(b) Cambios en prioridades del gasto}

La primera opción para un gobierno que busca aumentar el financiamiento de programas prioritarios debería ser reducir el gasto improductivo, particularmente aquellos de naturaleza recurrente. Esto puede requerir una revisión del gasto en temas tales como los subsidios existentes, transferencias para gasto corriente, cortes en el presupuesto de defensa y policía, ahorro en personal improductivo y reducción de la corrupción.

(c) Mayor eficiencia en el gasto

Espacio fiscal también puede ser creado por medio de mayor eficiencia en los gastos. Esto es aplicable, por ejemplo, en programas de educación (tasas mínimas maestro/alumno), salud (racionalización de los servicios de salud), asistencia técnica, entre otros.

\section{(d) Endeudamiento doméstico o extranjero}

La contratación de préstamos, ya sean domésticos o extranjeros, como mecanismo de creación de espacio fiscal, conllevan el compromiso de pago dentro de un cierto plazo. Por lo tanto, surge el punto si el retorno obtenido por el gasto compensa el costo del préstamo, o más directamente, si el gasto efectuado generará los ingresos necesarios para financiar el servicio de la deuda. En este sentido se tiene que evaluar el impacto de la nueva deuda sobre el nivel y servicio de la deuda existente; para esto se requiere considerar indicadores como el crecimiento futuro del PIB, de las exportaciones, de las remesas, los movimientos esperados de las tasas de interés, la elasticidad de los ingresos y la composición de la deuda actual.

\section{(e) El señoreaje del banco central}


Aunque el señoreaje de la banca central es generalmente entendido como la diferencia del valor de las monedas y billetes y el costo de producirlo, en el contexto de esta discusión se hace referencia a la capacidad de imprimir dinero para prestarlo al gobierno (Heller, 2005:3). Esta opción es la menos deseable y antes de asumirla debe evaluarse bien el impacto que tendría sobre la inflación.

\section{(f) Donaciones recibidas de fuentes extranjeras}

La posibilidad de que donaciones externas expandan el espacio fiscal debe descansar en la garantía previa de un flujo predecible y sostenido. O sea, que los países donantes se comprometan a sostener estas donaciones por un período específico, a modo de eliminar los cambios anuales que suelen presentarse. También es de considerar que grandes cantidades de donaciones pueden conducir a una apreciación del tipo de cambio, efecto éste que puede poner en riesgo el equilibrio del sector externo.

En resumen y según Heller, espacio fiscal puede ser creado siguiendo políticas macroeconómicas consistentes y efectivas. Estos espacios aumentan cuando los países tienen acuerdos con el FMI, dado que esto posibilita acceso a recursos externos, como los de apoyo presupuestario. También se insiste en la importancia de los temas que se derivan de la productividad del gasto, la sostenibilidad de la deuda o las implicaciones de solvencia de un programa de gastos de mediano plazo. Por lo tanto, es razonable a los donantes y a los países pedir al FMI evaluaciones objetivas y transparentes de los factores que determinan la sostenibilidad fiscal y de la deuda (Heller, 2005:14).

\subsection{El diamante del espacio fiscal y el enfoque del desarrollo}

El denominado diamante del espacio fiscal es una forma gráfica de mostrar las posibilidades u opciones macro fiscales para encontrar espacio fiscal bajo el marco de determinados objetivos de políticas públicas (Roy et. al., 2007:6). El diamante tiene cuatro pilares que son:

1) Asistencia Oficial del Desarrollo a través de ayuda y alivio de deuda

2) Movilización doméstica de recursos a través de reforma tributaria o mejoras de la administración tributaria

3) Financiamiento del déficit por medio de endeudamiento interno y externo

4) Reasignación y mayor eficiencia en el gasto.

Como se podrá apreciar en la Figura 1, el papel que juega cada uno de los componentes considerados es diferente por país. Por ejemplo, bajo determinadas circunstancias, la ayuda exterior puede ser crucial. En otros momentos, la movilización de recursos internos o el financiamiento a través del déficit pueden ser una mejor alternativa. Es por ello que los autores advierten el carácter específico, individual, del diamante de espacio fiscal para cada país.

Para la construcción del diamante de un país determinado es necesario definir los supuestos subyacentes de política, el período de tiempo implicado y el carácter endógeno o exógeno de las acciones de política para la generación de espacio fiscal. Lo anterior implica cinco pasos en la construcción del diamante, cuales son, clarificar los objetivos 
macroeconómicos y de desarrollo humano; identificar los desafíos fiscales de corto y largo plazo; identificar los desafíos exógenos y endógenos de corto plazo; construir el diamante; y, presentar el marco analítico completo (Roy et. al., 2007:6).

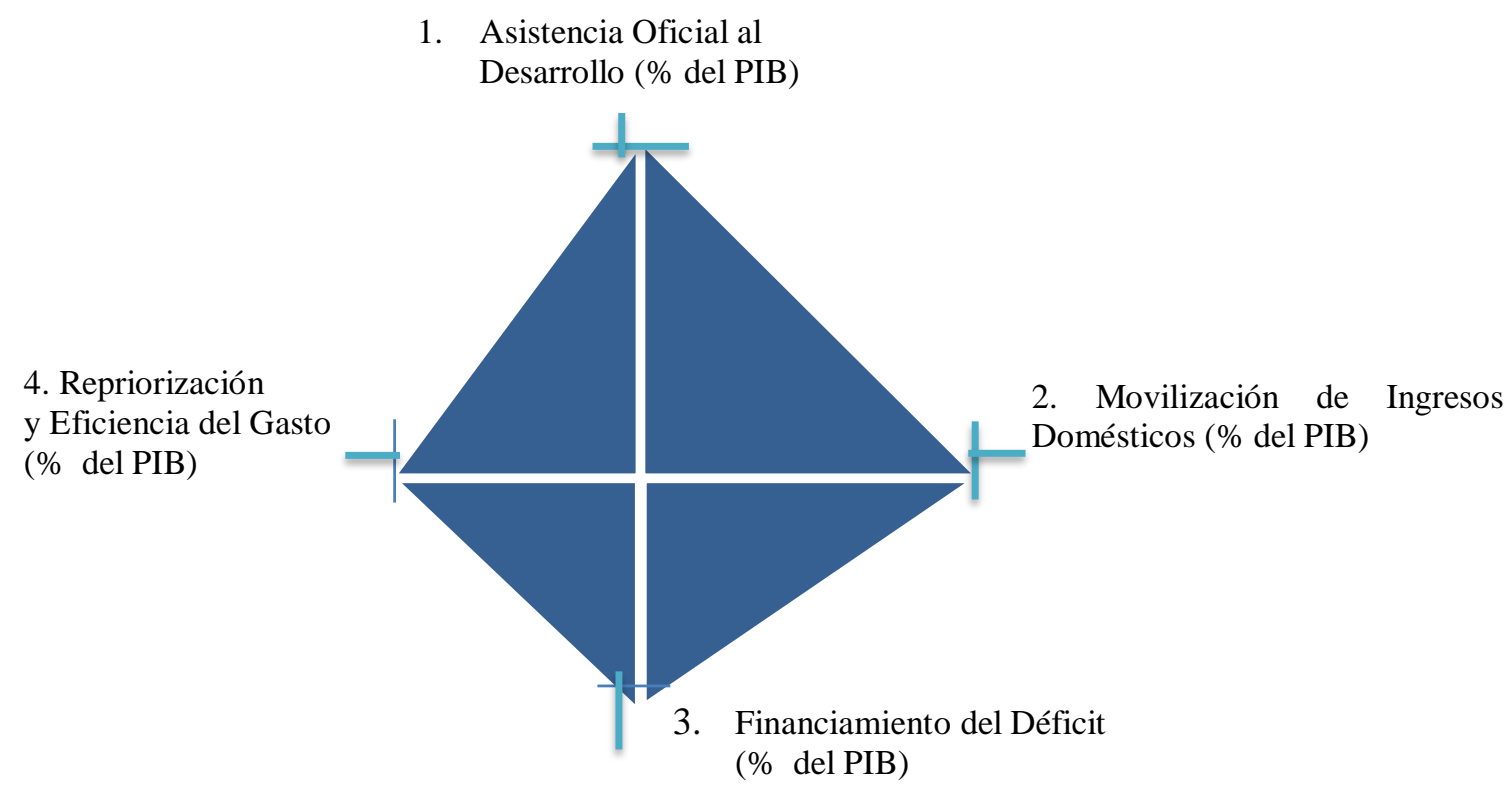

Figura 1. El diamante del espacio fiscal

Fuente: Roy, R., Heuty, A., \& Letouzé, E. (2007). Fiscal space for what? Analytical issues from a human development perspective. Istanbul: PNUD.

\subsection{Otras similitudes y diferencias entre los enfoques}

Como se podrá apreciar, las diferencias entre las fuentes de espacio fiscal no son menores. Por ejemplo, el enfoque de desarrollo no incluye el señoreaje, ni el enfoque de estabilidad enfatiza, aunque si lo asume, el ahorro proveniente de una mayor eficiencia en el gasto.

En materia de coincidencias, los dos enfoques tienen clara la inviabilidad de una estrategia sostenida de desarrollo basada únicamente en recursos externos. Esto implica decisiones no fáciles porque las administraciones de gobierno tienen un tiempo limitado, generalmente corto, de vigencia (aún en los países donde existe la reelección). Otra coincidencia es que ambos enfoques consideran el análisis del espacio fiscal como una tarea específica de cada país. Dicho análisis debe comenzar por una evaluación detallada de la posición fiscal inicial del país, la estructura de los ingresos y gastos públicos, las características de la deuda, la estructura económica, las perspectivas de obtención de recursos externos, así como el contexto externo que se enfrenta.

Para el enfoque de estabilidad y tratándose de la productividad o retorno del gasto en el mediano o largo plazo, sí éste altera la estabilidad financiera del momento, poco importará que sea corriente o de capital. Simplemente, tal gasto no debería efectuarse.

Sin embargo, el enfoque de desarrollo argumenta que la inversión social (o de infraestructura productiva) tiene sus réditos tanto para el crecimiento económico como para 
alcanzar objetivos sociales, y que esto no necesariamente implica alterar la sostenibilidad fiscal.

De otro modo, las propuestas de este segundo enfoque no son menos disciplinadas en el manejo de la política fiscal, pero sí muy diferentes y sensibles respecto a objetivos fiscales de largo plazo acordes con transformaciones más allá de la estabilidad fiscal. Esto expresa el conflicto entre el deseo de utilizar el gasto público para sacar a la población de la pobreza y la necesidad de un manejo fiscal prudente en términos del logro de determinadas metas macroeconómicas.

En general, la literatura sobre espacio fiscal adopta uno u otro de los enfoques arriba descritos. Por ejemplo, los trabajos de la OIT claramente consideran el objetivo del desarrollo humano sostenible como la principal motivación para la búsqueda de espacio fiscal (ver por ejemplo, Ortiz, Cummins, y Karunaneth 2015 y Duran-Valverde y Pacheco 2012). ${ }^{4}$ En los dos conocidos estudios de análisis de espacio fiscal en Honduras (Badillo, 2015 y Escobar, 2010), se soslaya la discusión sobre la conceptualización de espacio fiscal, pero es claro notar su orientación al enfoque de desarrollo. En los acuerdos de los países en desarrollo con el FMI implícitamente se asume el enfoque de estabilidad al privilegiar la búsqueda de equilibrios macroeconómicos sobre objetivos de mayor amplitud.

\subsection{Experiencias en la creación de espacio fiscal en países en desarrollo}

La mayoría de los países en desarrollo se caracterizan por los bajos presupuestos que destinan a la protección social. Sin embargo, existen experiencias internacionales positivas que muestran como estas limitaciones pueden superarse al asegurar el espacio fiscal apropiado. Durán-Valverde y Pacheco (2012) presentan la experiencia de ocho países en desarrollo en la creación de espacio fiscal. ${ }^{5}$ Estos investigadores lograron identificar en tal experiencia las siguientes fuentes de recursos fiscales: aumento general de impuestos; impuestos específicos a la explotación de recursos naturales; aumento de las contribuciones a la seguridad social; reasignación del gasto; reducción de la deuda pública; ayuda oficial al desarrollo; cambios en la legislación; mejoramiento de la eficiencia del gasto.

Como se podrá notar, las fuentes mencionadas forman parte del diamante descrito anteriormente, nada más que a un nivel más desagregado. Por ejemplo, los impuestos específicos sobre recursos naturales, aumento general de impuestos y aumentos de contribuciones a la seguridad social se podrían agrupar bajo la etiqueta de "Movilización doméstica de recursos", mientras que las otras dos fuentes formarían parte del grupo "Asistencia Oficial del Desarrollo a través de ayuda y alivio de deuda". Aparte de lo anterior, el aporte de Durán-Valverde y Pacheco es mostrar cómo los países, bajo determinado marco institucional y político, pueden impulsar medidas exitosas en la creación de espacio fiscal.

\footnotetext{
${ }^{4}$ El argumento que gastar en protección social no es posible se ha vuelto menos común en los foros sobre desarrollo internacional. Encontrar espacio fiscal para inversiones económicas y sociales críticas es necesario para el crecimiento inclusivo como para un Desarrollo humano sostenido, particularmente durante los tiempos malos (Ortiz, Cummins y Karunaneth, 2015:1).

${ }^{5}$ Bolivia, Botsuana, Brasil, Costa Rica, Lesoto, Namibia, Tailandia y Sudáfrica.
} 
El estudio en mención destaca catorce lecciones aprendidas, que por su importancia se presentan:

1) La estabilidad macroeconómica y la disciplina fiscal son esenciales para aumentar el espacio fiscal;

2) El crecimiento económico es importante, pero no es siempre el principal determinante en crear espacio fiscal para aumentar la protección social;

3) La voluntad política es fundamental;

4) El espacio fiscal para la extensión de la protección social es un asunto altamente político;

5) Los esquemas contributivos de protección social son un instrumento efectivo para generar espacio fiscal;

6) Las políticas de formalización del trabajo son un medio de crear espacio fiscal a través de la extensión de cobertura;

7) La renegociación de los términos de distribución de la riqueza generada por la explotación de recursos naturales es una opción factible;

8) La eficiencia en el manejo del gasto social sí importa;

9) El diseño de los sistemas de protección social también importa;

10) La inversión social paga y contribuye al desarrollo económico;

11) Hay ventajas en combinar programas contributivos con programas no contributivos;

12) Los beneficios de los programas no contributivos son una opción viable en la búsqueda de cobertura universal en países con una economía altamente informal;

13) El diálogo tripartito y la buena gobernanza son importantes para las contribuciones a la protección social;

14) Un esfuerzo sostenido y paciencia son elementos indispensables.

Como se puede apreciar, existe toda una riqueza de análisis y de acciones concretas en estas experiencias, que merecen tomarse en cuenta en los esfuerzos de creación de espacio fiscal de países como Honduras.

En la misma dirección, Ortíz, Cummins y Karunaneth (2015) sostienen que existen alternativas de financiamiento aún en los países más pobres para la protección social. En tal sentido analizan las experiencias de muchos países y muestran como, por ejemplo, Costa Rica y Tailandia reasignaron gasto militar para alcanzar servicios de salud universal. También se destacan casos como el de Brasil que usó un impuesto a las transacciones financieras para aumentar la cobertura de la protección social; Bolivia, Mongolia y Zambia utilizando impuestos sobre la explotación de sus recursos naturales para financiar pensiones y beneficios para la niñez; Argentina, Brasil, Túnez y Uruguay aumentaron las contribuciones de seguridad social. 
Los autores, además de incluir las fuentes de espacio fiscal ya analizadas, agregan otras a considerar. En general, señalan como fuentes de espacio fiscal: la reasignación del gasto público, el aumento de impuestos, la ampliación de cobertura seguridad social bajo esquemas contributivos, el cabildeo para el aumento de la ayuda al desarrollo, eliminación de los flujos financieros ilícitos, uso de las reservas fiscales e internacionales, préstamos o reestructuración de deuda, y adopción de un marco macroeconómico más flexible (Ortíz, Cummins y Karunaneth, 2015:1).

Con relación a las fuentes de espacio fiscal antes listadas, llama la atención la eliminación de flujos financieros ilícitos y el uso de reservas internacionales; los primeros incluyen los flujos del crimen organizado, pero también los que resultan de la evasión de impuestos y de la corrupción, aspectos bastante comunes en los países en desarrollo. Por su parte, el uso de reservas internacionales por arriba de lo establecido para enfrentar shocks externos, es una práctica novedosa descrita en el trabajo.

Con base al análisis realizado, Ortíz, Cummins y Karunaneth (2015) reiteran la advertencia de otros autores sobre la unicidad por país de las medidas para ampliar el espacio fiscal, y agregan, que las opciones deben analizarse con consideración a riesgos y compensaciones. De esta manera, las opciones serán el resultado de un diálogo social que permita promover el desarrollo socio-económico con empleo y protección social (Ortíz, Cummins y Karunaneth, 2015:56).

Es de agregar que desde la experiencia de la Iniciativa para los Países Altamente Endeudados (década pasada) hasta los Objetivos de Desarrollo del Milenio (1990-2015) y los Objetivos del Desarrollo Sostenible (2015-2030) se observa un respaldo, de hecho, al enfoque de desarrollo mediante la Asistencia Internacional al Desarrollo, misma que de cierta manera se dirige y condiciona al logro de objetivos de desarrollo resultantes de acuerdos globales. Muchos de tales objetivos son casi imposibles de alcanzar por muchos países de desarrollo bajo y medio sin el aumento sostenido del apoyo internacional. De esta manera toma más sentido la recomendación de Ortíz, Cummins y Karunaneth (2015) por acudir al cabildeo por el apoyo internacional, claro una vez demostrado el agotamiento de las posibilidades internas para financiar la protección social y otras metas de desarrollo.

\section{La búsqueda de espacio fiscal en Honduras}

\subsection{Esfuerzos previos}

El tema de espacio fiscal ha sido poco analizado en Honduras. Los dos trabajos conocidos hasta hace un par de años fueron los de Escobar (2010) y Badillo (2015). Escobar estableció que el objetivo de su trabajo era "identificar fuentes de financiamiento para que Honduras pueda aumentar la inversión pública en servicios sociales e infraestructura (2015:5)”. En otras palabras, definir el espacio fiscal en función de fuentes alternativas de financiamiento. De esta manera identificó tres fuentes de expansión de la inversión: aumento de los ingresos tributarios, reasignación del gasto y contratación de deuda. 
El análisis partió principalmente de la situación existente hasta $2008^{6}$, y de allí derivó recomendaciones como la reducción de las exoneraciones y mejoramiento de la administración tributaria desde el punto de vista de los ingresos. Mientras del lado del gasto, Escobar recomendó la reducción del peso de los salarios, la de los subsidios generalizados o mal focalizados y la contratación de deuda. Escobar estimó que la combinación de estas medidas podría producir recursos entre $4 \%$ y $6 \%$ del PIB. Adicionalmente, para que los recursos encontrados pudieran rendir frutos a futuro, las medidas deberían formar parte de un marco fiscal de mediano plazo. A su vez, este marco requeriría objetivos claros, como los Objetivos de Desarrollo del Milenio (ODM).

Los cambios propuestos no eran fáciles por lo que el autor aclaraba, "los países latinoamericanos que han logrado alterar elementos fundamentales de sus estructuras económicas de forma permanente, generalmente lo han logrado en el contexto de un acuerdo nacional" (Escobar 2010:33). Tal propósito requeriría, a su vez, transparencia y participación ciudadana.

Pese a que el análisis de Escobar se produjo en un contexto favorable para la economía hondureña y para sus finanzas públicas, varias de sus propuestas para ampliar el espacio fiscal mantienen vigencia en el contexto actual, aunque esté marcado por una situación de bajo crecimiento económico.

Por su parte, UNICEF-Honduras, con el apoyo técnico de Badillo (2015:1), también ha buscado identificar "recursos financieros existentes y potenciales, eficaces y sostenibles para la expansión y el fortalecimiento de la protección social; así como los marcos normativos y políticos para su sustentabilidad." Obviamente, el contexto macroeconómico para Badillo ya no es el mismo que sirvió de referencia a Escobar. ${ }^{7}$

El análisis de UNICEF-Honduras presenta tres escenarios, a saber, el inercial o de poca o ninguna intervención, que dejaría las finanzas públicas en deterioro continuo; el optimista, que asume un crecimiento del 6\% del PIB en los próximos años; y el del ajuste económico. UNICEF concluye que el ajuste económico es el camino adecuado, pero propone un abordaje integral de la fiscalidad, en simultaneidad y articulación con medidas sobre ingresos, gastos y financiamiento, y, además, un acuerdo nacional o pacto fiscal-social amplio, que otorgue legitimidad política a todas estas medidas.

La propuesta de UNICEF-Honduras es de ajuste, pero toma distancia de otros conocidos paquetes ortodoxos de austeridad. Se trata de un programa equitativo de reactivación social y económica, que por la gravedad de la situación fiscal hondureña analizada, requería de importantes rupturas con las políticas y prácticas del pasado, a lograr solamente sobre la base de un amplio acuerdo nacional (Badillo, 2015: 89). ${ }^{8}$

\footnotetext{
${ }^{6}$ El período de crecimiento económico más vigoroso de Honduras en las últimas décadas se produjo en el período 2004-2007.

${ }^{7}$ Los datos analizados por Badillo alcanzan en mayoría hasta 2013.

8 "En la coyuntura política actual, fortalecer el contrato social resulta de particular importancia en Honduras puesto que constituye el sostén del proceso de consolidación hacia el desarrollo humano y el crecimiento económico futuros, y la política fiscal, la piedra angular de un nuevo contrato social o de un nuevo pacto de convivencia para el futuro Gobierno, en el que unos impuestos justos y eficaces se reconozcan explícitamente como el sustrato de la provisión de un gasto público de alta calidad" (Badillo, 2015:77).
} 
Sobre lo anterior dos consideraciones devienen importantes. La primera, que precisamente la ruta de la austeridad ortodoxa fue la que el país siguió bajo un acuerdo de tres años con el FMI. Ello agravó el carácter regresivo de la estructura tributaria y aumentó las distorsiones en la asignación del gasto. La segunda, que el Grupo Promotor del Diálogo Fiscal y la Alianza por un Pacto Fiscal en Honduras han propuesto públicamente un pacto fiscal. Sin embargo, la actual administración de gobierno se ha limitado a la escucha pasiva.

Badillo (2015) también analizó la disponibilidad de recursos fiscales en función de las siguientes variables: el tamaño de la economía (medido por el PIB y su crecimiento), la normativa tributaria y su capacidad de implementación (evasión y elusión fiscal, exoneraciones y eficiencia de la administración tributaria, entre otros), y el nivel de eficacia y eficiencia del gasto público. De este análisis, el autor derivó una serie de recomendaciones en el campo de los ingresos, gastos y financiamiento. Algunas de estas medidas son retomadas en nuestra propia propuesta. Como se podrá apreciar, los trabajos de Escobar (2010) y Badillo (2015), con pocas diferencias, arriban a conclusiones similares sobre las posibilidades de encontrar espacio fiscal en Honduras.

\subsection{La situación fiscal actual y la protección social}

La búsqueda de fuentes de espacio fiscal en la Honduras actual enfrenta el desafío de un proceso de consolidación fiscal reciente. Aun así, nunca está de más la discusión y la búsqueda de alternativas encaminadas a ampliar el espacio fiscal para la protección social y el desarrollo humano.

Para algunas teorías el crecimiento económico vigoroso bastaría para erradicar la pobreza. Sin embargo, la realidad se muestra compleja y multicausal, así que los casos concretos enseñan que el derrame de bienestar solo llega a los más pobres cuando el crecimiento económico se refuerza con políticas públicas adecuadas que garanticen empleo, y otras modalidades de distribución del ingreso y protección social.

Buena cantidad de la población más pobre sufre niveles tan bajos de competencias laborales, que su inserción al mercado de trabajo deviene imposible, al menos se le brinden las oportunidades apropiadas para sobrevivir y mejorar su situación; en algunos casos, muchos hogares necesitan más de una generación para asegurar las competencias que se demandan. Además, las eventualidades del ciclo de vida suelen ocurrirle a cualquiera, tal es el caso de los accidentes, las enfermedades, los desastres naturales, la pérdida del empleo, la vejez, la muerte del o los proveedores del hogar, etc. La protección social integral deviene un instrumento complementario poderoso para distribuir (el bienestar) y anticipar el riesgo a caer en la pobreza (ILO, 2010; OIT, 2011; Sen y Dreze, 2011). ${ }^{9}$

\footnotetext{
9 “La crisis actual una vez más ha demostrado cuán importante es el papel que juega la protección/seguridad social en una sociedad en tiempos de crisis y ajuste. Esta actúa como un irremplazable estabilizador económico, social y político tanto para las vidas individuales como la vida de la sociedad en su conjunto. La seguridad social juega este papel en adición a otras funciones, como proveer mecanismos para aliviar y prevenir pobreza, para reducir disparidades de ingreso a niveles aceptables, y también para mejorar el capital humano y la productividad. La seguridad social es, entonces, una de las condiciones para el desarrollo económico y social sostenible. Es un factor en el desarrollo. Es también un importante factor en el Estado y sociedad modernos y democráticos". (ILO, 2010:5; traducción libre). En términos más generales, Sen y Dreze han mostrado que "El crecimiento, por supuesto, puede ser muy útil para lograr el desarrollo, pero esto
} 
La situación fiscal de Honduras en la actualidad resulta el producto de dos acontecimientos simultáneos iniciados en 2009. Por una parte, la gran recesión internacional que frenó el ritmo de crecimiento que el país venía experimentando. Por otra, el golpe de Estado de mediados de ese año y la polarización política que desencadenó y, finalmente, impactó en la asignación de recursos públicos. Con relación al primer aspecto, el PIB de Honduras había aumentado a una tasa promedio de 6\% entre 2004 y 2007, una expansión extraordinaria en décadas; sin embargo, en los años subsiguientes la tasa de crecimiento promedio fue tan solo de 3.5\%. Estudios del Banco Mundial (2015) y BID (2017) establecen que este bajo nivel de la actividad económica continuará en los próximos años, hecho que limitará las posibilidades de mayores recursos fiscales.

Por otra parte, la crisis política condujo a resultados que facilitaron la concentración de poder, lo que a su vez derivó en un aumento de los déficits fiscales por motivos electorales. De esta forma, el déficit del gobierno central se mantuvo arriba del $4.5 \%$ en 2010 y 2011 , para alcanzar $6 \%$ y $7.9 \%$ en 2012 y 2013 , respectivamente. Lo anterior requirió un proceso de ajuste fiscal derivado de la firma de un acuerdo con el FMI a partir de 2014 y con una vigencia de 3 años, que finalizará en diciembre 2017. El acuerdo incluyó las principales medidas de reducción del déficit como son el aumento de impuestos (mayoritariamente indirectos) y recortes del gasto público.

El proceso de ajuste ha tenido resultados positivos al bajar el déficit fiscal de $7.9 \%$ del PIB en 2013 a $2.8 \%$ en 2016. No obstante, ha sido un logro con cargo al aumento de la regresividad del sistema tributario y la reducción de la oferta de bienes públicos como educación y salud. ${ }^{10}$

Pese a la reducción del déficit, la deuda pública aumentó de $25 \%$ del PIB en 2009 a 45\% en 2016, representando el monto total de la deuda el $240 \%$ de los ingresos fiscales de este año. La acumulación acelerada de deuda produjo un fuerte aumento del servicio de la deuda, de tal forma que para 2017 representa más de dos veces el presupuesto de salud y más de una vez el presupuesto de educación.

El proceso de ajuste fiscal elevó los impuestos y mejoró la administración tributaria del país. De esta manera la carga tributaria pasó de $14.8 \%$ en 2013 a 18.0\% del PIB en 2016. Durante los últimos tres años se han impulsado medidas administrativas contra la evasión de impuestos como la factura electrónica, el fortalecimiento de la unidad de grandes contribuyentes y otros.

Adicionalmente, para reforzar el proceso de consolidación fiscal se aprobó, en 2016, la Ley de Responsabilidad Fiscal que establece topes al gasto corriente y al déficit público. No obstante, estos avances, y a medida que se acercan las elecciones de noviembre de 2017, se han presentado retrocesos en la administración tributaria, entre ellos la aprobación del nuevo código tributario. El nuevo marco legal sustituye la renta universal como cálculo para el impuesto sobre los ingresos por la renta territorial, aun cuando es bastante conocido que la última se presta más para el movimiento de flujos financieros ilícitos a nivel

requiere de políticas públicas activas para garantizar que los beneficios del crecimiento económico sean ampliamente compartidos" (Sen y Dreze. 2011:1).

${ }^{10}$ Para un detalle de las medidas adoptadas durante el ajuste véase Icefi (2017). 
internacional. Adicionalmente se despenalizó el delito tributario, medida que torna más difícil el cobro para la autoridad recaudadora.

Por otra parte, la estructura del gasto ha cambiado notablemente y, con ello, ha puesto de manifiesto las prioridades reales de las dos últimas administraciones gubernamentales. $\mathrm{Al}$ 2010, las mayores cifras del presupuesto del gobierno central pertenecieron a educación, salud y servicios públicos generales, éste último compuesto principalmente de transferencias para gasto corriente del Congreso Nacional y la Corte Suprema de Justicia. Al 2017, la prioridad la ocupan los pagos por el servicio de la deuda pública, educación, servicios públicos generales y defensa y seguridad. No obstante, como se aprecia en el Cuadro 1, la educación se muestra como prioridad decreciente al haber bajado en casi 13 puntos porcentuales, de $32.8 \%$ en 2010 a $20.0 \%$ en 2017. También ha descendido la prioridad de la salud como componente del presupuesto público, de $14.7 \%$ a $10.8 \%$.

El descenso del gasto en educación se relaciona con las reformas al Estatuto Docente, que lograron controlar el incremento de los sueldos de los docentes. Sin embargo, persiste en educación la escasa atención a las inversiones en la expansión y mejoramiento de las instalaciones, laboratorios, equipos y re-entrenamiento (actualización) de docentes.

Por su parte, el descenso presupuestario en salud ha significado aumentar la carga para los hogares en los gastos respectivos. En 2011, un estudio poco difundido de la Secretaría de Salud (SESAL et al. 2011) estimó que del gasto total en salud (8.6\% del PIB), la mitad fue financiado por los hogares. Esto implicó, entre otros hechos, que $76 \%$ de los gastos en medicamentos corrieron con cargo a los hogares. Tal participación de los hogares conmueve por ser de las más altas del mundo. Siendo que respecto a 2011 el presupuesto al sector salud se ha reducido, es de esperar que la situación arriba descrita se habrá empeorado.

Tabla 1. Honduras: Estructura del presupuesto de egresos de la administración central por finalidad (porcentajes sobre el total del gasto)

\begin{tabular}{l|cc}
\multicolumn{1}{c}{ CONCEPTO } & 2010 & 2017 \\
\hline Servicios públicos generales & $14.0 \%$ & $16.9 \%$ \\
Defensa y seguridad & $\mathbf{1 1 . 7 \%}$ & $\mathbf{1 3 . 5 \%}$ \\
Asuntos económicos & $10.5 \%$ & $9.4 \%$ \\
Protección del medio ambiente & $1.1 \%$ & $0.5 \%$ \\
Vivienda y servicios comunitarios & $0.9 \%$ & $0.2 \%$ \\
Servicios de salud & $14.7 \%$ & $10.8 \%$ \\
Educación, investigación, cultura y actividades & $32.8 \%$ & $20.0 \%$ \\
Protección social & $\mathbf{3 . 4 \%}$ & $\mathbf{6 . 6 \%}$ \\
Deuda pública & $\mathbf{1 0 . 9 \%}$ & $\mathbf{2 2 . 0 \%}$ \\
TOTAL & $100.0 \%$ & $100.0 \%$
\end{tabular}

Fuente: Secretaría de Finanzas, 2017. 
En suma, el gran perdedor del cambio de prioridades presupuestarias es el gasto social, a excepción de lo ocurrido con la asistencia social (el Programa Vida Mejor), que se incrementó en 3.2 puntos porcentuales. En todo caso, la protección social en general (asistencia social más seguridad social) nunca ha sido prioridad presupuestaria, en seguimiento a una tendencia generalizada en América Latina, una región que solo aparece por encima de África en materia de atención presupuestaria a la protección social. Ver Recuadro 1.

Así que Honduras enfrenta para los próximos años un panorama de bajo crecimiento (entre $3 \%$ y 4\%), una carga tributaria recientemente aumentada y notoriamente regresiva, resultados mixtos en la administración de los tributos, y un gasto social venido a menos entre las prioridades del gasto público. Estos son los puntos de partida a tener en cuenta en la búsqueda de espacio fiscal para los objetivos del desarrollo humano sostenible que el país se ha propuesto alcanzar en los próximos años.

\section{Recuadro 1. Gasto en protección social en perspectiva comparativa}

De acuerdo al Grupo Banco Mundial (2015:49), el gasto en protección social y empleo de Honduras como porcentaje del PIB en 2013 ocupaba el segundo lugar en la región (7.0\% frente a 8.7 de Costa Rica y 6.2\% de Panamá). Ese 7.0\%, se compuso de los gastos en seguridad social (1.6\%) y asistencia social y empleo (5.4\%). El promedio del gasto en protección social para América Latina al 2013 podría haber rondado entre 8\% a 9\% del PIB.

Un estudio reciente de la CEPAL (Sojo. 2017:78) da a conocer los resultados del estudio de Ocampo y Gómez-Arteaga (2016) que construyeron un índice multidimensional de protección social con base a ocho indicadores relacionados a cobertura de distintos servicios. De acuerdo a dicho índice (basado en datos CEPAL 2013 y 2015), Honduras aparece dentro del grupo de países con "protección social limitada" y en el último lugar de América Latina. Los indicadores utilizados para construir el índice fueron: cobertura de pensiones contributivas, afiliación de asalariados a sistemas de pensiones y a sistemas de salud, brecha del acceso a pensiones de asalariados y no asalariados, brecha del acceso a sistemas de salud de asalariados y no asalariados, acceso de hogares pobres a algún tipo de programa social, cobertura de asistencia social del quintil más pobre, gasto en protección social como porcentaje del PIB y gasto social en salud como porcentaje del PIB.

Al cambiar la comparación de gasto con relación al PIB a gasto per cápita, el panorama para Honduras se muestra mucho más desolador. Se carece de una comparación actualizada, pero con base a cifras de 2009-2010, el Panorama Social de América Latina y el Caribe 2012 de la CEPAL (Anexo Estadístico, Cuadro 48) mostraba igualmente a Honduras en el último lugar, con un gasto de tan solo 11 US dólares frente a USD476 como promedio ponderado para América Latina y el Caribe. Ecuador aparece como el país con el gasto más cercano a Honduras (USD61). En Centroamérica el Salvador mostraba un gasto de USD128, Panamá USD229, Costa Rica USD340, Uruguay USD796 (el más alto de todos los países de la lista). Se trata de US dólares de 2005. 


\section{Espacio fiscal para aumentar la protección social en Honduras: Una propuesta.}

Honduras aparece en un estudio reciente en el último lugar de América Latina y dentro del grupo con "protección social limitada" (Ver Recuadro 1). Esto obliga a analizar con renovado interés el espacio fiscal que sea posible crear para el mejoramiento de la protección social en el país. La tarea es difícil, dado que Honduras enfrentará un panorama de bajo crecimiento económico para los próximos años (entre 3\% y 4\%), una carga tributaria aumentada en los últimos años y altamente regresiva, y una administración tributaria con resultados mixtos. Sin embargo, existen caminos que recorrer para cerrar estas brechas.

Basado en los trabajos anteriores sobre Honduras y en el análisis sobre la situación fiscal actual se presentan algunos elementos a considerar en la búsqueda de espacio fiscal. Para ello, si se utiliza el diamante fiscal (Heuty y Letouzé, 2007) con datos recientes del país se lograría la Figura 2, sobre la cual se pueden hacer las siguientes consideraciones:

4. Repriorización y Eficiencia del Gasto (22.9\% del PIB)

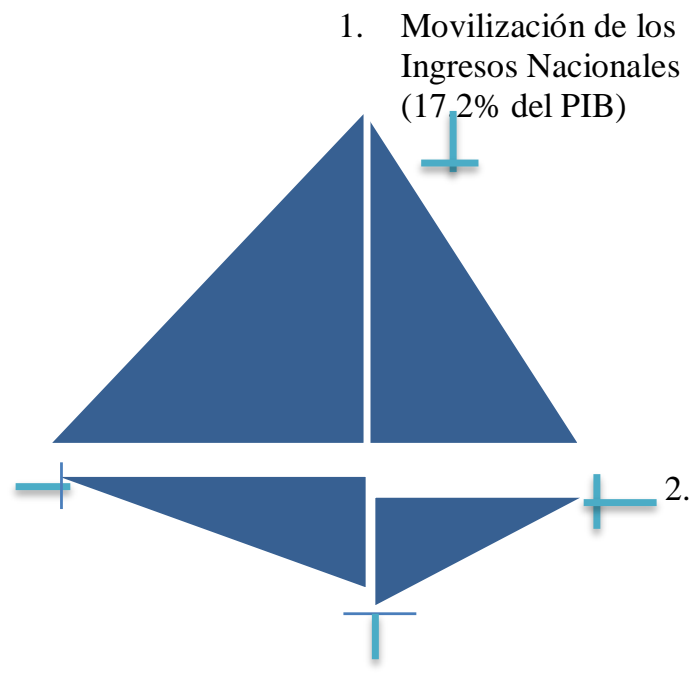

2. Asistencia Oficial al Desarrollo (3.2\% del PIB $)^{11}$

3. Financiamiento del Déficit (3.4\% del PIB)

Figura 2. Honduras: Diamante de Política Fiscal (promedio 2014-2016)

Fuente: Roy, Heuty, Letouzé (2007) con datos del Banco Mundial y la Sefin.

- En los últimos tres años, la carga tributaria, sin incluir seguridad social, ha representado en promedio $17.2 \%$ del PIB (Ver Figura 2). Si bien es una de las cargas más altas de la región, datos de países como Argentina y Brasil $(24.0 \%$ y 23.7\% del PIB en 2015, respectivamente) sugieren que todavía puede haber un margen para negociar dicha carga. Adicionalmente, los países antes mencionados destinan importantes recursos para la seguridad social: $7.1 \%$ del PIB en el caso de

11 Promedio de 2013-2015. 
Argentina y $8.3 \%$ del PIB en el caso de Brasil. Esto contrasta con el 3\% del PIB que en el caso de Honduras se recauda para el mismo fin (OCDE/CEPAL/CIAT/BID, 2017:151).

- La posibilidad de un aumento de las tasas tributarias no debería descartarse, pero es importante que tal negociación ocurra dentro del marco de un pacto fiscal, para asegurar que prevalezca el sentido de reciprocidad entre las autoridades gubernamentales y la ciudadanía. A corto plazo, también se podrá encontrar espacio fiscal en la parte tributaria al reducir las exoneraciones fiscales, que ya no se justifican. De acuerdo a algunos estudios estas exoneraciones representan entre el $5 \%$ y $7 \%$ del PIB. Por otra parte, la reducción de la evasión fiscal también ayudará a liberar recursos. La ventaja de estas medidas es que podrán financiar gasto recurrente. Esto requiere un fortalecimiento de la administración tributaria, que ha tenido avances y retrocesos en los años recientes.

- Una oportunidad que se presentará en el futuro cercano es la aprobación del Monotributo, régimen tributario particular establecido en el nuevo Código Tributario vigente desde el 1 de enero de 2017. La entrada en vigencia del Mono-tributo depende de la aprobación de una ley especial que se espera sea aprobada en 2018. La oportunidad se presenta no solo para ampliar la base tributaria, sino también la cobertura de la seguridad social, bajo el entendido de una correcta aplicación de incentivos.

- La asistencia oficial al desarrollo difícilmente podrá considerarse una fuente de espacio fiscal en Honduras por varios motivos:

(a) El primero, porque Honduras recibió a mediados de la década anterior una condonación de deuda de parte de los organismos financieros internacionales que difícilmente se repetirá en el futuro próximo.

(b) Segundo, porque la asistencia al desarrollo ha venido disminuyendo en la región centroamericana en los últimos años; para Honduras representa en promedio en los últimos tres años un $3.5 \%$ del PIB.

(c) Tercero, porque la estructura de la deuda se modificado hacia préstamos en condiciones de mercado, que reduce el grado de concesionalidad. El país, por lo tanto, tiene que destinar más recursos para el pago de servicio de deuda. Sin embargo, el país debía comprometerse seriamente con metas de transparencia, de equidad presupuestaria y de eficiencia del gasto para poder justificar su clamor por mayor apoyo internacional en la forma de ayudas y alivio de deuda, en tanto necesario para alcanzar en tiempo los Objetivos del Desarrollo Sostenible en materia de protección social y reducción de pobreza. ${ }^{12}$

- Con relación a la deuda pública (tanto interna, como externa), dado su fuerte incremento, pareciera que no existe espacio fiscal para seguirse endeudando, al menos en el corto plazo. No obstante, en el mediano plazo la deuda para proyectos

12 El Ejercicio prospectivo de la incidencia de la Ley Marco del Sistema de Protección Social en el presupuesto público y en la reducción de la pobreza extrema en Honduras, publicado por el Instituto de Investigación de Políticas Públicas de UNITEC (Díaz, W., \& Marín, C., 2017), concluye que los recursos necesitados para darle cumplimiento a la mencionada Ley y para alcanzar los objetivos gubernamentales de reducción de pobreza al 2030 son de tal magnitud que resulta muy difícil para el país el pretender tales logros. 
de infraestructura y gasto social podría ser considerada. Se debe recordar que la deuda que se convierte en carga es aquella que en el mediano plazo no apoya el crecimiento y la competitividad del país. Tanto el mejoramiento de la infraestructura económica como social pueden alcanzar estos resultados. En este sentido, es de considerar que la nueva Ley de Responsabilidad Fiscal, si bien es positiva para la disciplina fiscal, también puede constituirse en una camisa de fuerza para alcanzar objetivos de desarrollo.

- La re-priorización del gasto y el aumento de su eficiencia parecen ser mecanismos idóneos para encontrar espacio fiscal para el financiamiento de la protección social. En efecto, el análisis preliminar muestra que el énfasis en los últimos años ha estado en los gastos para el servicio de la deuda pública, en seguridad y defensa, y en servicios públicos generales; estos gastos podrían reorientarse. Lo que no se puede dejar de lado son las implicaciones políticas de dichas decisiones y el hecho de que ahorros sustanciales procedentes de mejorar la eficiencia del gasto público toma tiempo.

Organizaciones como el Grupo del Banco Mundial (2015) ha mostrado áreas objetivo para el propósito de eficiencia del gasto. También el BID señaló alternativas para re-direccionar el gasto con fines de eficiencia en aspectos relacionados a la protección social, tales como la mejora de la focalización y de la coordinación de los programas y la mayor participación de los municipios en la operación, e incluso financiamiento, de los programas de protección social (BID, 2014:31).

- Entonces, aunque se requiere profundizar y discutir sobre los mecanismos específicos de espacio fiscal para la protección social, se observan caminos ya analizados y por los que se puede transitar; la parte más difícil está en encontrar acuerdos nacionales (pacto fiscal y social) que permitan viabilizar esas opciones técnicas.

- Por otra parte, quedan varios desafíos pendientes. Por una parte, está el de alcanzar un alto grado de consenso nacional sobre el modelo y extensión de la protección social a la que se aspira, lo mismo que de la necesidad de prever recursos suficientes para financiar y volver sostenible no solo la protección social sino también otros compromisos de país relacionados con los Objetivos del Desarrollo Sostenible. Por otra, se requiere abrir la mente a diferentes modalidades innovadoras para ensanchar el espacio fiscal. Esto demandará el acopio de información sobre mecanismos empleados en diferentes países, así como la evaluación de su viabilidad en Honduras.

\section{Bibliografía}

Badillo, Daniel. (2015). Estudio sobre espacio fiscal para la protección social de la niñez y adolescencia en Honduras. Unicef-Honduras. Tegucigalpa.

Díaz, W., \& Marín, C. (2017). ¿Cuánto costará? Ejercicio prospectivo de la incidencia de la Ley Marco del Sistema de Protección Social en el presupuesto público y en la reducción de la 
pobreza extrema en Honduras. Tegucigalpa. Serie Avances de Investigación. Instituto de Investigación de Políticas Públicas (IIPP)-UNITEC.

Duran-Valverde, F., \& Pacheco, J.F. (2012). Fiscal space and the extension of social protection: Lessons learnt from developing countries. Extension of Social Security (ESS) Paper No.33 (Geneva, International Labor Organization)

Escobar, L. (2010). Honduras: espacio fiscal para la inversión social y productiva. Tegucigalpa. PNUD-Honduras. Obtenido de

http://www.undp.org/content/dam/honduras/docs/publicaciones/HN_042010.pdf

Grupo del Banco Mundial. (2015). Honduras. Estudio de gasto público social y sus instituciones (educación, salud, protección social y empleo). Washington.

Heller, Peter. (2005). Understanding fiscal space. International Monetary Fund. Policy Discussion Papers (PDP 05/04).

International Labour Organization. (2010). World Social Security Report 2010-11: Providing coverage in times of crisis and beyond. Geneva. ILO.

Instituto Centroamericano de Estudios Fiscales (Icefi). (2015). Honduras: Una política fiscal que no mejora el presente ni construye el futuro. Análisis del Proyecto de Presupuesto de Ingresos y Egresos de la República 2016. Tegucigalpa. Obtenido de http://icefi.org/publicaciones/honduras-una-politica-fiscal-que-no-mejora-el-presente-niconstruye-el-futuro-0

Icefi. (2017). La política fiscal reciente en Honduras (2013-2016). Editorial Guaymuras.

OCDE/CEPAL/CIAT/BID. (2017). Estadísticas tributarias en América Latina y el Caribe 19902015. Paris. OCDE Publishing.

Organización Internacional del Trabajo (OIT). (2011). Piso de Protección Social para una globalización equitativa e inclusiva. Informe del Grupo consultivo sobre el Piso de Protección Social. Ginebra, Organización Internacional del Trabajo

Ortiz, I., Cummins, M., \& Karunaneth, K. (2015). Fiscal Space for Social Protection. Options to Expand Social Investments in 187 Countries. Extension of Social Security (ESS) Working Paper No.48 (Geneva, International Labor Organization).

Roy, R., Heuty, A. \& Letouzé, E. (2007). Fiscal Space for What? Analytical Issues from a Human Development Perspective. G-8 Workshop on Fiscal Policy. Istanbul.

Sen, A., \& Dreze, J. (2011). Putting Growth in its Place. Outlook (November).

SESAL-USAID-ULAT-INE-OPS/OMS. (2011). Estudio de Gasto y Financiamiento en Salud. Tegucigalpa. 